\title{
Journey from Infertile Frame to Fertile Living
}

\author{
Ramya $S^{*}$
}

\section{ABSTRACT}

Longing for children and the heartbreak of futility have been a part and parcel of life since the beginning of mankind. The paper gives an in depth understanding of infertility as a medical, psychological and social condition with suitable theoretical frameworks. The paper identifies the key role of infertility counselor, a mental health professional trained in reproductive medicine, in an individual/couple journey from the beginning of treatment to beyond treatment. Infertility counselor contributes by providing effective therapeutic interventions to improve patient wellbeing and response to treatment and giving different perspectives for understanding the issue from cultural, gender and societal point of view. Finally, the paper discusses importance of the transition from childlessness to child-free and the role of infertility counselor in facilitating it.

Keywords: Infertility Treatment, Infertility counseling, Theoretical frameworks, Psychological interventions, Transition to child-free

\section{INTRODUCTION}

Infertility is defined as an inability to conceive after 12 months of unprotected sexual intercourse. Primary infertility refers to the failure to conceive by a couple who has never conceived, while secondary infertility refers to the failure to conceive by a couple who had previously conceived (Sharon, Linda, 2006, pg 20). The World Health Organization (WHO) estimates that 60 to 80 million couples worldwide currently suffer from infertility (WHO, 2004). In a country like India pivoting varied traditions and cultures where womanhood is considered to be complete only by childbearing, the national prevalence of infertility is between 7 and 10 percent and prevalence of primary and secondary infertility are 3 and 8 percent respectively (WHO). According to Multi-centric study conducted by WHO, primary and secondary infertility rates are 3 and 8 percent respectively. Though there is wide variance in incidence rates of infertility among different countries, the toll it takes on individuals emotional and social wellbeing results in significant and unique psychosocial consequences.

\section{Interpreting Infertility}

There are various models that interpret infertility and its causal factors. In this paper medical model, psychological models and social construct theory are discussed particularly.

\footnotetext{
*Psychological Counselor, Cloudnine Fertility, 250, 24th Main, 5th Main, JP Nagar (Opp. Nandini Hotel) Bangalore-78

(C) 2014 Ramya S; licensee IJIP. This is an Open Access Research distributed under the terms of the Creative Commons Attribution License (http://creativecommons.org/licenses/by/2.0), which permits unrestricted use, distribution, and reproduction in any Medium, provided the original work is properly cited.
} 


\section{Medical Model}

Infertility is commonly identified as an illness, mostly a chronic illness, since it is long term, is shrouded with uncertainty and can dominate and intrude one's life. The medical model of infertility attributes pathology to biological and molecular processes (Mc Daniel, Hapworth and Doherty 1992). In approximately $90 \%$ of the cases referred for infertility treatment, a physical reason for the infertility can be diagnosed (Greil 1991, Monarch 1993).

The major causes of female infertility include (1) failure to ovulate, (2) structural or functional abnormalities of the Fallopian tubes, (3) endometriosis or adhesions in the pelvis of the woman that interfere with capture of the egg by the Fallopian tube, (4) poor timing or technique of intercourse, (5) infections of the reproductive tract, (6) immunological barriers to fertilization or implantation, and (7) an abnormal uterine lining that may interfere with implantation of the embryo.

The major causes of male infertility (pathological causes) can be broadly classified as pretesticular, testicular and post-testicular. Some pre-testicular causes of infertility are hypo- and hyper gonad tropic hypogonadism, Kallman syndrome and medications or genetic abnormalities that affect the hypothalamic-pituitary-gonadal (HPG) axis. Common testicular causes of infertility are varicocele, cryptorchidism, testicular injury, testicular cancer and congenital abnormalities. Examples of post testicular causes of infertility are congenital bilateral absence of the vas deferens (CBVAD), erectile dysfunction, Young's Syndrome, nerve injury and abnormal coital practices.

\section{Full Psychogenic Infertility Model and Psychological consequence model}

Both of these models contrast each other. According to Full Psychogenic Infertility Model all cases of sterility and infertility are basically caused by psychological problems or mechanisms. Psychological disturbances of various kinds are perceived as generating infertility. Physical obstructions were considered as secondary causes. For example, Folds and Folds (1979) described tubal occlusion as generated by spasms or temporary occlusions that, in turn had a psychogenic origin. Where as in Psychological consequence model causality is reversed. It is assumed that infertility -rather than being caused by psychological factors- may instead lead to psychological problems, such as depression, stress, anxiety, etc., A study by Klonoff- Cohen revealed a strong evidence supporting the notion that psychological factor such as stress effects pregnancy outcomes where in they found subjects who expressed lowest baseline of distress were $93 \%$ more likely to have a live birth when compared to subjects who reported highest baseline level of distress levels. However some studies ended with opposite results. Though these causal factors are contradictory and debatable, the aspect psychological counseling remains

important. In a study (Domar et al; Boston IVF, Harvard Medical School) 43\% of couples who 
underwent counseling, achieved pregnancy compared to $16.7 \%$ in the control group, which shows the effectiveness of psychological interventions in the outcome of infertility treatment.

\section{SOCIAL CONSTRUCT THEORY}

From the prospective of social construct theory, infertility is not a static condition with predictable psychosocial outcomes, but rather a dynamic, social conditioned process that changes individuals struggle to deal with and to make meaning of their unique experience of infertility. Often the process of constructing a meaning about infertility begins once a couple suspects a problem, even before medical treatment is sought (Greil, Leitko, Porter, 1988). This theory is critical in understanding how different people interpret infertility. It addresses cultural, religious, and environmental factors (e.g., natural or man-made disasters such as hurricanes or terrorist attacks) that can and do intensify or somehow influence the infertility experience for individuals and couples.

\section{Remedial measures to Infertility}

Almost every society has culturally approved solutions to infertility - social, spiritual, and medical -Divorce, polygamy, and extramarital affairs remain various forms of adoption and fostering, as they have long been, social solutions to infertility. Since antiquity, the appeal of religious faith and the power of belief in spirits and gods as a remedy for infertility can be found in all cultures. Various forms of medical approaches which the older generation has tried and gone become the foundations for new innovations spurting in the science arena. For example, the tales that suggest that the ancients were familiar with the 'idea' of artificial insemination, one story states that "God Shiva once spurted semen when he saw Vishnu in the form of the celestial enchantress Mohini. Sages collected this and gave it to the wind-god Vayu, who poured it into the 'ear' (a common mythical metaphor for the womb) of Anjani, a monkey, who gave birth to Hanuman, the monkey-god. Devoid of the mythical aura, one might say that the tale refers to the practice of artificial insemination: semen is transferred to the womb without sexual intercourse). And the other story in the Bhagvata Purana, which suggests the practice of surrogate motherhood. Kans, the wicked king of Mathura, had imprisoned his sister Devaki and her husband Vasudeva because oracles had informed him that her child would be his killer. Every time she delivered a child, he smashed its head on the floor. He killed six children. When the seventh child was conceived, the gods intervened. They summoned the goddess Yogamaya and had her transfer the fetus from the womb of Devaki to the womb of Rohini (Vasudeva's other wife who lived with her sister Yashoda across the river Yamuna, in the village of cowherds at Gokul). Thus the child conceived in one womb was incubated in and delivered through another womb."

And these days with the advancement in technology that opens horizons infertility issues are systematically dealt beginning from screening to a wide variety of treatment options that are available with inventions in pharmacology. For women, few screening techniques employed are 
Hormone testing (Anti Mullerian Hormone AMH, follicle stimulating hormone FSH, Luteinizing hormone LH), Sonographic parameters for Ovulation testing (like Antral follicle count, Ovarian Volume and ovarian Vascular Flow), uterus evaluation methods like Hysterosalpingography (HSG), Ultrasound, Sonohysterography, and Hysteroscopy, endometrial biopsy (which tests the lining of the uterus), tubal patency tests like HSG, Saline infusion Sonography, Laparoscopic Chromotubation and Fluoroscopic Tubal Cannulation, Pap smear to check for signs of infection, postcoital test to identify cervical causes, and Special X-ray tests. For men, Sperm testing through semen analysis and Testicular biopsy (surgical extraction of sperm). And procedural techniques employed to treat Infertility are like IUI, IVF and ICSI. Intra-Uterine Insemination (IUI) with or without controlled ovarian hyper stimulation $(\mathrm{COH})$ is a cost effective fertility treatment that involves placing a number of washed sperm directly into the uterus. The rationale of IUI is to reduce the effect of factors such as vaginal acidity and cervical mucus hostility and to benefit from the deposition of a bolus of concentrated motile morphologically normal sperms as close as possible to oocytes. In vitro fertilization (IVF) refers to a procedure where the woman's eggs are retrieved by planned and timed trigger and fertilized with the man's sperm outside the body, in a laboratory and Intra Cytoplasmic Sperm Injection (ICSI) is an advanced fertilization method where a single sperm is injected directly into the egg using a fine glass needle. The embryos, thus formed are then placed back into the uterus to achieve a pregnancy (Embryo Transfer ET) Today, India amidst moral, ethical and religious constraints, has become the hub of Fertility Clinics providing flexible treatment with different options for couples seeking infertility treatment ranging from IUI (own sample or donor sample), IVF self cycle to third party reproduction like IVF donor oocyte Cycle, donor sperm cycle, donor embryo cycle, and surrogacy. However, this flexibility is bound by its own constraints like age, finance, success rate, etc along with unidentifiable psychogenic factors which might increase the uncertainty of the treatment.

\section{Theoretical frameworks that convey life beyond the treatment boundary}

Although the world encountered a great deal of changes from civilizations, societal structures to advancement in science and technology since the beginning of creation, childbearing remain fundamentally important though the value and reasons for having children may have changed.

According to family systems theory, the family life cycle framework defines a series of stages with expectable timelines that most people imagine as their predictable life course. Successful passage through family life cycle stages depends on the effectiveness of developmentally appropriate negotiations of tasks and stressors. Carter and McGoldrick delineated six stages of the American family life cycle, a model that acknowledges the confluence of situational, developmental, and family-of-origin (historical) stressors. The stages and their tasks are:1. Unattached young adult, 2. Married couple, 3. Family with young children 4. Family with adolescents 5. Parents launching children 6. Family in later life. While Parenthood enables couples moving forward through the predictable stages of the family life cycle childlessness 
stops the whole family system dynamics. In short, childless couples trapped in the 'couple stage' of the family life cycle can feel lost in a painful 'no man's land' of quasi-adulthood, on the fringe of family flux, where individual, couple, and family roles are ill defined and/or uncomfortable while at the same time feeling responsible for preventing other family members and/or the family system from moving forward. According to R. Matthews and A. Matthews the transition to nonparenthood is as important and demanding a transition for families and individuals as the more traditional transition to parenthood. The childless couple must redefine the meaning of family to include a marital dyad without children. Couples must alter their life, goals, and personal identities and all members of the family system must adapt and adjust as they rework family boundaries these are challenging tasks for individuals, couples, and families.

Erickson identified eight stages of life critical in human psychosocial development. While they parallel family development, the focus of Erickson's stages is on individual development and tasks that balance the negative and positives in each developmental stage. The stages most pertinent to involuntarily childless men and women are: young adulthood: Intimacy versus isolation; adulthood: Generativity versus self-absorption; and senescence: Integrity versus disgust. As Erickson pointed out, failure to move through this developmental stage leads to a "pervading sense of stagnation and interpersonal impoverishment" potentially impacting healthy adjustment later in life and successful achievement of developmental tasks contributes to happiness and success in later tasks. Developmental tasks are based on physical maturation; cultural pressures; and privileges and include the aspirations and values of the individual. Erickson's generative task of midlife is an excellent example of how the successful accomplishment of a developmental task need not be restricted to reproduction (as is very often the socially and culturally defined norm). Generativity can also expand beyond childbearing to include a multitude of creative endeavors; mentoring possibilities; and nurturing opportunities, allowing the mature adult to guide and influence the next generation.

Ireland suggested a variation of object-relations and Lacanian theory to better understand the woman who is not a mother. "Unlike the male, who must reject his early identification with mother and shift his identification to father, the daughter's identity evolves through a path of continual relatedness; she will never have to completely relinquish her earliest maternal identification." Consequently, the desire or need for recreating the mother-child bond is intimately tied to a woman's identity formation and maturation. Unfortunately, because the childless woman identifies with her mother, it is difficult for her to see herself in the roles of competency and independence beyond the role of motherhood. Simply put: All mothers are women, but not all women are mothers. The mother who models a multifaceted personality extending beyond her 'good' or 'bad' mothering offers her daughter more opportunity to identify with and be influenced by her mother's personality and creative expression of identity. Motherhood is no longer the one and only defining component of a woman. Ireland contended that personal identity based on an expanded view of femaleness better enables a woman to develop her full potential 
The danger of childlessness due to voluntary delay, or ongoing, but unsuccessful medical treatment, lies in the possibility of never making the decision to live without children. Endless treatment options can keep the wound open and delay healing. Year after year, grief persists and festers. Fulfillment and happiness become as elusive as parenthood. The focus of a man or woman's identity becomes a sense of not having, not belonging, and not sharing. The cost of unresolved grief can be the additional losses of relationships, marriage, jobs, career, and other life plans. There is also a substantial risk of depression, anxiety, and other mental and physical health difficulties.

As it's clearly evident that Infertility is not just a medical condition, but also a social and emotional condition, its crucial to adopt infertility counseling as a part of infertility treatment to provide a holistic approach. Infertility counseling as an emerging specialty within the mental health professions, has gained recognition and respect for its professional contributions through patient care, research, and education as well as for the identification of the need for expert care and treatment of this unique population in conjunction with complex medical treatment. Infertility counselor refers to any mental health professional who has special training in reproductive medicine alongside competency in assessments and therapeutic interventions. The Infertility counselor evaluates, diagnoses, and treats psychological disorders that emerge as couple confronts infertility. Since infertility is an emotional turmoil for the couple from the time of initial diagnosis to treatment to post infertility, and nature of treatment itself being a mental block adding on to the stress, infertility counselor takes charge of addressing these issues by providing patient education, an arena for facilitating decision making, a forum for discussing ethical and cultural issues related to treatment, and emotional follow-up when the treatment results in a pregnancy and especially, when it does not.

\section{JOURNEY FROM CHILDLESSNESS TO BE CHILD-FREE}

"Choosing to be child-free after infertility is not giving up hope, it is finding hope of a good life again, only this time without children." -Carter and Carter

Opting to live child-free could be a result of fruitless journey through varied infertility treatments discussed above or adaptation for couples when childlessness is the only alternative. Child-free is a hopeful word used to describe the positive potential in life? To live child-free is not resigning oneself to a life without children, rather a conscious, deliberate decision to define oneself with a new identity, set new goals and the ability to invest renewed energies into work, family, and hobbies. To live child-free is a choice requiring clarity of thoughts, feelings, and communication before becoming acceptable to both partners. This reengineering of self and family is an active process in which living child-free is a way to fulfill the goals of parenthood in other positive, constructive ways. People with the desire to nurture and love should be encouraged to find ways to do this without having children. The move to a child-free life is a creative adaptation from 
sorrow, pain, and loss.

However this major transition is difficult if the couple has not been able to come to an agreement on being child-free and in such cases infertility counseling recommended. Through various means of therapeutic intervention infertility counselors can help individuals and couples process their losses and then, in time, guide decision making and assist their transformation of identity in pursuit of new life meaning. Ultimately, the goal is to help the client consciously accept and find fulfillment and safety in their new and unexpected life journey. In this, confronting loss is the first step wherein one must face the loss of the dreamed-of child; the loss of whatever part of one's identity was wrapped up in those dreams; and the loss of a destiny that one always assumed would be achieved. Shapiro contended that: "Couples can't rein in control over their emotions and private lives until they cease their quest for a baby." Deciding to stop treatment can be more devastating than being on a treatment treadmill with the variety of treatment options available today. Medical intervention locks the couple into a cycle of hope, followed by the crushing despair of failure. They often feel they cannot give up and think that if they keep trying, eventually something will work. Only the honest and realistic acceptance of their circumstances allows their hope and energy to return.By facilitating the grief and loss, as the mourning process is over, psychological well being is restored out of denials and emotional paralysis.

Facilitating decision making is the second step in moving towards being child-free where in the counselor plays a role in clearing barriers of communication gap and helping one to express himself/herself in an effective way. Compromise is the main goal, but may not be possible if there are difference of opinions between couple where in one is ready to accept new changes and the other desires to be a parent, also if childlessness is culturally unacceptable. By giving the opportunity to verbalize their feelings and helping them appreciate each other's views and by renewing their commitment to each other, possible solutions can emerge. Generally, client(s) and infertility counselor agree to a time-limited contract with clearly defined goals. Typically, goals will be met within two to ten sessions. After years of riding on emotional roller coaster, possibilities of feeling helpless or denial of the situation might peep in, if left unworked they have the potential of leading to clinical depression. Using solution-focused therapy or a cognitive-behavioral framework, infertility counselors can help clients reconnect with their feelings in a safe and supportive environment using various strategies like Self-instructions that include Distraction, Imagery, Motivational self-talk and further leading to the development of adaptive coping strategies for minimizing negative or self-defeating thoughts and changing maladaptive beliefs. Another therapy, which is found effective is Rational-Emotive Behaviour Therapy (REBT) by Albert Ellis proposes a 'biopsychosocial' explanation of causation - i.e. That a combination of biological, psychological, and social factors are involved in the way humans feel and act. 'ABC' theory of personality that illustrates the role of cognition is central to REBT Theory and Practice. In this framework ' $A$ ' represents an actual event or experience, and the person's 'inferences' or interpretations as to what is happening like "I am not able to reproduce". 'B' represents the 'evaluative' beliefs that follow from these inferences "I am not 
complete". 'C' represents the Consequences, emotions and behaviours that follow from those evaluative beliefs like "Victimizing isolating one". A does not give rise to C, instead, B largely causes $\mathrm{C}$. Ellis adds D and E to ABC: The therapist must dispute (D) the irrational beliefs (whole aspect of you is not identified only by childbearing, there are many aspects that attributes to life), in order for the client to ultimately enjoy the positive psychological effects (E) of rational beliefs (Feeling good about oneself there by rechanneling the energy in a constructive way to other aspects of life). Developing Insights about ones irrational beliefs and working on eliminating it and Unconditional Acceptance of Self, Others and Life are key techniques for major transition to occur.

The basic goal of using REBT in Infertility set up is to assist clients in minimizing emotional disturbances and self defeating thoughts and behaviours, and by the end of session become more self actualized so that they can lead a happier existence. The therapist leaves the client with self help techniques such as self observation and personal change that will help them maintain their gains and enable coping in the long-term future.

For clients suffering from long-standing depression or dysthymia, a thorough assessment may lead to a referral and further evaluation of pharmacological intervention in conjunction with longer-term, insight-focused and interpersonal psychotherapy. As the depressive symptomatology recedes, work in therapy typically returns to active decision making within an outcome-focused framework. As stated earlier, confronting loss is the first step in deciding to remain child-free. Support groups can do great job where in telling one's story offers an opportunity to identify losses. This group of people with a common issue or situation utilizes the sense of community to share stories and helpful hints, give/receive support (emotional), promote services and help care for each other where in spending time with other couples in the same position makes them feel less vulnerable and victimized. Patients network with each other and help support each other and further the Counselor facilitates the sessions. Sessions are not limited to indoor counseling, they can go beyond where in creative expression such as drawing, painting, poetry, or collage can be helpful in facilitating bereavement and moving on and for some creating a ritual or ceremony to say 'good-bye' to the dream child might help in acknowledging the loss and to move further.

Basic strategies for decision making involve taking control of one's life; developing a positive identity; and reasserting goals and priorities. Rubin offers a very useful model for helping clients with decision making. Assessment starts with looking for and identifying possible 'decision blocks': (1) resignation or holding on to pain; (2) believing there is only one correct decision; (3) holding on to all possible options rather than choosing any one path; (4) procrastination; and (5) guilt over previous decisions. The work in therapy is to identify the individual or couple's particular obstacles and, through the use of therapeutic interventions, overcome these blocks to allow the process of decision making to unfold. According to Rubin's model, the next task is to establish a foundation for decision making based on personal values. When consumed by the blindfold of infertility, the perception and definition of self become myopic. It is suggested that 
either during or between sessions, the clients take the time to create a list of priorities. Possible items on the list might be health, quality of life, religion, education, culture, family influence, and security. As clients develop a list, they see themselves as full human beings with the ability to alter their behavior to match their values and create or follow other life plans not involving parenthood. The final task entails committing to the decision. This means operationalising the decision to stop treatment and moving from being childless or the state of having less to the position of choosing a child-free life. For most couples, it is a gradual process that happens over time. They may find it takes months or even years before being child-free feels right or comfortable. Rarely do partners move through this process in the same way and with the same timeline. Allowances should be made for individual and gender specific differences. Eventually, everything begins to fall into place, and concrete evidence of moving on appears. Perhaps a house is purchased without a large family room and four bedrooms, or an application to graduate school is completed or a career move is made. For others, it might be new hobbies; meaningful volunteer work; new friends; or a renewed focus and commitment to their marriage. Realistically, for some couples moving on may be doing so separately because the meaning and purpose of marriage (to have children) has been nullified by childlessness. In these instances, the infertility counselor's work may involve facilitating an amicable marital dissolution; helping both or either partner grieve the lost spouse as well as child; and helping to explore other life goals at possibilities.

\section{CONCLUSION}

Infertility could be a result of any cause, but its consequences cost, high on one's emotional and social well being. Infertility has the potential to threaten one's financial well being, intimacy between couple and relationship with family members and society. But how much ever distressful it could be, it is a condition that has occurred at a particular stage of life. Nobody grows up being infertile, we grow up giving many meanings to life and identifying ourselves with it. At one stage of life if the identification was with the parenthood and if its not achieved after several trials, it does not mean life ends there. Stagnation would only increase the burden. Life has to move on no matter what condition an individual is in. Like how Epictetus articulated "Men are disturbed not by events, but by the views which they take of them", changing one's perception of infertility and life events is very important to redefine goals and move forward in life. Emphasis on psychological services should be promoted by health care organizations to avail the service for the betterment of patient's condition. Mental health professionals should actively take responsibilities in rendering such services. Alongside, this movement not only be restricted to clinical team and the patient, since infertility is a social stigma, to eradicate this, participation of each member of a society is as essential, to re-frame one's cognitions about infertility, as it is to a patient. 


\section{BIBLIOGRAPHY}

1. Bliss C (1999), The Social Construction of Infertility by Minority Women. (Doctoral Dissertation) Retrieved from http://gerrystahl.net/personal/family/dissertation.pdf

2. Carter JW, Carter M (1989), Sweet Grapes: How to Stop Being Infertile and Start Living Again, USA, Perspectives Press.

3. Corey Gerald(2012), Theory and Practice of Counselling and Psychotherapy ( $^{\text {th }}$ edition), USA Cengage Learning. Retrieved from http://books.google.co.in/

4. Dr Greg Mulhauser, An Introduction to Rational Emotive Behaviour Therapy (Online Article) Retrieved from http://counsellingresource.com/lib/therapy/types/rationalemotive/

5. Erickson E.(1980) Identity and the Life Cycle. New York, Norton.

6. Froggatt Wayne (2005), A Brief Introduction To Rational Emotive Behaviour Therapy ( $3^{\text {rd }}$ edition). Retrieved from http://www.rational.org.nz/prof-docs/Intro-REBT.pdf

7. Froggatt Wayne (2005), A Brief Introduction To Rational Emotive Behaviour Therapy ( $3^{\text {rd }}$ edition). Retrieved from http://www.rational.org.nz/prof-docs/Intro-REBT.pdf

8. Ginsburg S Elizabeth (2012), In Vitro Fertilization: A Comprehensive Guide, New York, Springer publications. Retrieved from http://books.google.co.in

9. Goldman B Marlene et al (2000), Women and Health ( $2^{\text {nd }}$ edition), California, Academic press. Retrieved from http://books.google.co.in

10. Hammer Burns, L. And Covington, S. N. (2006) Infertility Counselling: A Comprehensive Handbook for Clinicians ( $2^{\text {nd }}$ edition) London, Cambridge University Press.

11. Herson Michel(2002), "Encyclopedia of Psychotherapy", Academic Press, USA.

12. Inhorn M C, Balen FV.,(2002) Infertility around the Globe: New thinking on Childlessness, Gender and Reproductive Technologies, California, University of California Press Ltd.

13. Jessie PN (2013), Psychosocial Aspects of Infertility, Indian Journal of Applied Research, 3 (8), 634-636.

14. Meniru G I (1997), A handbook of Intrauterine Insemination, UK, Cambridge University press. Retrieved from http://books.google.co.in

15. Nene UA, Coyaji K, Apte H (2005), Infertility: a label of choice in the case of sexually dysfunctional couples, Patient Education and Counseling, 59 (3), 234-8. Retrieved from http://www.sciencedirect.com/science/article/pii/S0738399105002429

16. Pattanaik D (2014, March $22^{\text {nd }}$ ), 'Infertility, Artificial Insemination and Surrogate Mother in Hindu Mythology' ( Online Blog Post ) Retrieved from http://blog.drmalpani.com/2014/03/infertility-in-hindu-mythology-dr.html

17. Plessis Du S S, Agarwal A, Sabanegh E S,(2014) Male Infertility: A Complete Guide to Lifestyle and Environmental Factors, New york, Springer Publishers. Retrieved from http://books.google.co.in/

18. Rao K A, et al (2014), Principles and Practice of Assisted Reproductive Technology, New Delhi, India, Jaypee Brothers Medical Publishers (P) Ltd.

19. Sharf R S (2010), Theories of Psychotherapy and Counselling: Concepts and Cases (5th edition), Belmont, USA, Cengage Learning . ( $p$ p 339). Retrieved from http://books.google.co.in/ 


\section{WEBS:}

20. http://india.unfpa.org/drive/fertility.pdf

21. http://www.ncbi.nlm.nih.gov/pmc/articles/PMC2569858/

22. http://www.rebtnetwork.org/whatis.html 